\title{
About finite energy solutions in thermoelasticity of micropolar bodies with voids
}

\author{
Marin Marin ${ }^{* *}$, Adina Chirila ${ }^{1}$, Andreas Öchsner ${ }^{2}$ and Sorin Vlase ${ }^{3}$
}

\section{"Correspondence:}

m.marin@unitbv.ro

1 Department of Mathematics and

Computer Science, Transilvania

University of Brasov, Brasov,

Romania

Full list of author information is

available at the end of the article

\begin{abstract}
Our study is dedicated to the problem with initial and boundary conditions in the theory of thermoelasticity for micropolar materials with pores. We obtain some results regarding the existence and uniqueness of a finite energy solution of this mixed problem by generalizing the corresponding results obtained by Dafermos in the context of classical elasticity. In some specific conditions we prove some properties regarding the possibility to control the finite energy solution.
\end{abstract}

MSC: 74A15; 74F05; 35Q74; 35Q79

Keywords: Micopolar bodies; Voids; Finite energy solution; Existence of solution

\section{Introduction}

It is known that a material that is under the action of high temperatures is influenced by a heat flow which will involve a temperature distribution, and this will give rise to thermal stresses. The role of the pertinent material properties can affect the magnitude of thermal stress, so it must be well understood and all possible modes of failure must be considered. Taking into account that our study approaches the materials with voids, we can hope that our results can help the specialists in applications in which some materials with voids are involved, such as in the production of granular materials or geological layers. It should be underlined that the granular theory of Goodman and Cowin in [1] gave the start of research on materials with voids. This theory together with the approach of Cowin and Nunziato in [2] has a characteristic feature: the presence of some small pores in a model of a classic solid which are considered by adding to each particle an additional degree of freedom. Considering the two theories of materials with voids, this degree of freedom will be used to characterize the mechanical evolution of granular solids. In this case the pores of the material are interstices and the material of matrix is elastic.

The important implications of this theory are related to manufactured porous objects, such as ceramics, pressed powders, and also to geological layers, such as rocks or soil. In the initial stage, the approaches of Cowin and Nunziato were applied only to solids which are non-conductor of heat (see also [3]). Then, the theory was extended to cover thermoelasticity of the materials with voids. So, the paper of Iesan [4] is a straightforward extension for solids for which the thermal effect is considered.

(c) The Author(s) 2019. This article is distributed under the terms of the Creative Commons Attribution 4.0 International License (http://creativecommons.org/licenses/by/4.0/), which permits unrestricted use, distribution, and reproduction in any medium, provided you give appropriate credit to the original author(s) and the source, provide a link to the Creative Commons license, and indicate if changes were made. 
On the other hand, in our work we take into account the micropolar structure of the materials. This is part of a more general concept, that of the microstructure, which includes a large number of extensions of classical elasticity theory such as micromorphic structure, microstretch structure, Cosserat structure, dipolar structure, microtemperatures, and so on. The start in microstructure theories was given by Eringen (see $[5,6])$. Then, the interest in these theories has grown enormously, as evidenced by a huge number of studies published in this context. Here we list a few of them, such as [7-24].

In our study we will extend the result obtained by Fichera in [25] and Dafermos [26] regarding the existence of solutions in the context of linear elasticity theory. Here, the asymptotic stability for the finite energy solution is also approached. We must say, however, that the results of Dafermos, in turn, are based on the results of Visik [27].

The strategy of our work is the following. First, we put down the main equations and conditions characteristic to the mixed problem for the theory of the micropolar thermoelastic materials having voids. Then, we introduce the notion of finite energy solutions and show a way to expand the set of finite energy solutions. The result regarding the uniqueness of this kind of solution is obtained for the general case of non-homogeneous conditions and boundary data. We first obtain an existence result regarding a solution with finite energy, and this is deduced regarding the null initial data. Then, this result is extended to the general non-homogeneous initial conditions. In our last result we deduce several estimations useful to control the evolution of the finite energy solution.

\section{Basic equations}

We assume that our thermoelastic micropolar body with voids occupies at the moment $t=0$ a regular domain $D$ from the space $R^{3}$, the usual Euclidian space. Denote by $\partial D$ the border of $D$ and assume it to be a sufficiently regular surface, at least to admit the application of the divergence theorem. The closure of $D$ is denoted by $\bar{D}$. The motion of our medium will be reported to the rectangular axes $O x_{i},(i=1,2,3)$. The usual vector and tensor notations are adopted. We will use a superposed dot to designate the time derivative of a function, while a partial derivative with respect to a spatial variable is denoted by a subscript preceded by a comma. In the case of repeated indices, the Einstein summation is used. When there is no risk of confusion, the time argument and/or the spatial argument and that of a function will be omitted.

The mass density $\varrho$, the volume fraction $\nu$, and the density of matrix material $\gamma$ in the reference configuration are represented by

$$
\varrho_{1}=\gamma_{1} v_{1}
$$

Here, $\gamma_{1}, v_{1}$ depend only on the time variable, not on the spatial variables.

In order to describe the motion of a thermoelastic micropolar body with voids, we will use the following independent variables:

- $u_{i}(x, t)$-the displacement vector field from the reference configuration;

- $\varphi_{i}(x, t)$-the vector for the micopolar displacement;

- $\theta$-the difference between the current temperature and $T_{0}$, that is,

$$
\theta=T-T_{0}
$$


- $\phi$-the difference between the current volume fraction temperature and $v_{1}$, that is,

$$
\phi=v-v_{1} .
$$

Using the above variables $u_{i}(x, t), \varphi_{i}(x, t)$, we will introduce the components of the tensors of strain, namely $\varepsilon_{i j}$ and $\gamma_{i j}$, as follows:

$$
\varepsilon_{i j}=u_{j, i}+\varepsilon_{i j k} \varphi_{k}, \quad \gamma_{i j}=\varphi_{j, i}
$$

We assume that the body is free of initial stress and couple stress and has zero intrinsic equilibrated body forces and zero flux rate. Being in the context of linear theory, it is natural to consider that the internal energy density is a quadratic form with the following expression:

$$
\begin{aligned}
\varrho_{0} e= & \frac{1}{2} A_{i j m n} \varepsilon_{i j} \varepsilon_{m n}+B_{i j m n} \varepsilon_{i j} \gamma_{m n}+\frac{1}{2} C_{i j m n} \gamma_{i j} \gamma_{m n} \\
& +d_{i j k} \varepsilon_{i j} \phi_{, k}+e_{i j k} \mu_{i j} \phi_{, k}-\frac{1}{2} a \theta^{2}-a_{i} \phi_{, i} \theta \\
& -\alpha_{i j} \varepsilon_{i j} \theta-\beta_{i j} \gamma_{i j} \theta+\frac{1}{2} a_{i j} \phi_{, i} \phi_{, j}+\frac{1}{2} K_{i j} \theta_{, i} \theta_{j,} .
\end{aligned}
$$

We can use a technique based on the proposal by Nunziato and Cowin in paper [2]. So, taking into account that

$$
t_{i j}=\frac{\partial e}{\partial \varepsilon_{i j}}, \quad m_{i j}=\frac{\partial e}{\partial \gamma_{i j}}, \quad h_{i}=\frac{\partial e}{\partial \phi_{, i}}, \quad \eta=-\frac{\partial e}{\partial \theta}, \quad q_{i}=\frac{\partial e}{\partial \theta_{, i}},
$$

one can obtain the connections between the strain tensors and the stress tensors, that is, the constitutive equations:

$$
\begin{aligned}
& t_{i j}=A_{i j m n} \varepsilon_{m n}+B_{i j m n} \gamma_{m n}+d_{i j k} \phi_{, k}-\alpha_{i j} \theta, \\
& m_{i j}=B_{i j m n} \varepsilon_{m n}+C_{i j m n} \gamma_{m n}+e_{i j k} \phi_{, k}-\beta_{i j} \theta, \\
& h_{i}=D_{m n i} \varepsilon_{m n}+E_{m n i} \gamma_{m n}+a_{i j} \phi_{, j}-a_{i} \theta, \\
& \varrho \eta=\alpha_{i j} \varepsilon_{i j}+\beta_{i j} \gamma_{i j}+a_{i} \phi_{, i}+a \theta, \\
& q_{i}=K_{i j} \theta_{, j} .
\end{aligned}
$$

Also, we can deduce the following fundamental equations (see also [23]):

- the motion equations:

$$
\begin{aligned}
& t_{i j, j}+\varrho f_{i}=\varrho \ddot{u}_{i}, \\
& m_{i j, j}+\varepsilon_{i j k} t_{j k}+\varrho g_{i}=I_{i j} \ddot{\varphi}_{j} ;
\end{aligned}
$$

- the equations of the equilibrated forces:

$$
h_{i, i}+\varrho l=\varrho \kappa \ddot{\phi}
$$


- the equation of evolution of energy:

$$
\varrho T_{0} \dot{\eta}=q_{i, i}+\varrho r
$$

The notations used in the previous equations have the following significance:

- $\varrho$-the density of mass;

- $\eta$-the entropy specific to the body;

- $T_{0}$-the constant temperature in the initial state;

- $\kappa$-the balancing inertia;

- $\varepsilon_{i j}, \gamma_{i j}$-the tensors of strain;

- $t_{i j}, m_{i j}$-the tensors of stress;

- $h_{i}$-a stress vector for equilibrium;

- $q_{i}$-a heat flux vector;

- $f_{i}, g_{i}, l$-body forces;

- $r$-the supply of heat;

The functions $A_{i j m n}, B_{i j m n}, C_{i j m n}, d_{i j k}, e_{i j k}, \alpha_{i j}, \beta_{i j}, a_{i j}, a_{i}, a, K_{i j}$ characterize the elastic properties of the body and satisfy the following symmetries:

$$
A_{i j m n}=A_{m n i j}, \quad C_{i j m n}=C_{m n i j}, \quad K_{i j}=K_{j i} .
$$

In our work we approach an inhomogeneous material which is supposed to be anisotropic.

From the Clausius-Duhem inequality, that is, the inequality of entropy production, we can deduce that the thermal conductivity tensor $K_{i j}$ is positive semidefinite, that is:

$$
K_{i j} \theta_{i} \theta_{, j} \geq 0 .
$$

We can observe that the basic Eqs. (4) and (6) are similar to those from the classical theory of thermoelasticity. The new equations of the equilibrated forces (5) are motivated in the paper [28] by a variational approach (see also [29]).

For completing the mixed problem in the theory of thermoelastic porous bodies, we have to prescribe some initial data and some boundary conditions. The initial data are given for $t=0$ :

$$
\begin{array}{lll}
u_{i}(0)=u_{i}^{0}, & \dot{u}_{i}(0)=u_{i}^{1}, & \text { in } \bar{B}, \\
\varphi_{i}(0)=\varphi_{i}^{0}, & \dot{\varphi}_{i}(0)=\varphi_{i}^{1}, & \text { in } \bar{B}, \\
\phi(0)=\phi^{0}, & \dot{\phi}(0)=\phi^{1}, & \text { in } \bar{B}, \\
\theta(0)=\theta^{0}, & \text { in } \bar{B} .
\end{array}
$$

Also, we will use the boundary data, given for $t \in\left[0, t_{0}\right)$, as follows:

$$
\begin{array}{lll}
u_{i}=\bar{u}_{i} \quad \text { on } \partial D_{1}, & t_{i}=\bar{t}_{i} \quad \text { on } \partial D_{1}^{c}, \\
\varphi_{i}=\bar{\varphi}_{i} \quad \text { on } \partial D_{2}, & m_{i}=\bar{m}_{i} \quad \text { on } \partial D_{2}^{c}, \\
\phi=\bar{\phi} & \text { on } \partial D_{3}, & h=\bar{h} \quad \text { on } \partial B_{3}^{c}, \\
\theta=\bar{\theta}_{i} & \text { on } \partial D_{4}, \quad q=\bar{q} \quad \text { on } \partial D_{4}^{c} .
\end{array}
$$


Here, $t_{i} \equiv t_{i j} n_{j}, m_{i} \equiv m_{i j} n_{j}, h \equiv h_{i} n_{i}, q \equiv q_{i} n_{i}$ and $n=\left(n_{i}\right)$ is the unit normal outward oriented of the surface $\partial D$. The time $t_{0}$ can be $\infty$. The surfaces $\partial D_{1}, \partial D_{2}, \partial D_{3}, \partial D_{4}$ and respectively $\partial D_{1}^{c}, \partial D_{2}^{c}, \partial D_{3}^{c}, \partial D_{4}^{c}$ are parts of the surface $\partial D$, such that

$$
\begin{aligned}
& \partial D_{1} \cup \partial D_{1}^{c}=\partial D_{2} \cup \partial D_{2}^{c}=\partial D_{3} \cup \partial D_{3}^{c}=\partial D_{4} \cup \partial D_{4}^{c}=\partial D, \\
& \partial D_{1} \cap \partial D_{1}^{c}=\partial D_{2} \cap \partial D_{2}^{c}=\partial D_{3} \cap \partial D_{3}^{c}=\partial D_{4} \cap \partial D_{4}^{c}=\emptyset .
\end{aligned}
$$

Also, $u_{i}^{0}, u_{i}^{1}, \varphi_{i}^{0}, \varphi_{i}^{1}, \theta^{0}, \phi^{0}, \phi^{1}, \bar{u}_{i}, \bar{t}_{i}, \bar{\varphi}_{i}, \bar{m}_{i}, \bar{\phi}, \bar{\theta}, \bar{q}$, and $\bar{h}$ are given and regular functions.

If we take into account Eq. (4), then the basic Eqs. (1), (2), and (3) receive the following form:

$$
\begin{aligned}
& \ddot{u}_{i}= \frac{1}{\varrho}\left[\left(A_{i j m n} \varepsilon_{m n}\right)_{, j}+\left(B_{i j m n} \gamma_{m n}\right)_{, j}+\left(d_{i j k} \phi_{, k}\right)_{, j}-\left(\alpha_{i j} \theta\right)_{, j}\right]+f_{i}, \\
& \ddot{\varphi}_{i}= \frac{1}{I_{i j}}\left[\left(B_{i j m n} \varepsilon_{m n}\right)_{, j}+\left(C_{i j m n} \gamma_{m n}\right)_{, j}+\left(e_{i j k} \phi_{, k}\right)_{, j}-\left(\beta_{i j} \theta\right)_{, j}\right. \\
&\left.+\varepsilon_{i j k}\left(A_{j k m n} \varepsilon_{m n}+B_{j k m n} \gamma_{m n}+d_{j k m} \phi_{, m}-\alpha_{j k} \theta\right)+g_{i}\right], \\
& \ddot{\phi}= \frac{1}{\varrho \kappa}\left[\left(D_{m n i} \varepsilon_{m n}\right)_{, i}+\left(E_{m n i} \gamma_{m n}\right)_{, i}+\left(a_{i j} \phi_{, j}\right)_{, i}-\left(a_{i} \theta\right)_{, i}\right]+\frac{1}{\kappa} l, \\
& \alpha_{i j} \dot{\varepsilon}_{i j}+\beta_{i j} \dot{\gamma}_{i j}+a_{i} \dot{\phi}_{, i}+a \dot{\theta}=\frac{\varrho}{T_{0}}\left(K_{i j} \theta_{, j}\right)_{, i}+\frac{1}{T_{0}} r .
\end{aligned}
$$

The array $\left(u_{i}, \varphi_{i}, \phi, \theta\right)$ is called the solution of the mixed problem in the theory of thermoelastic micropolar materials with pores for any $(x, t) \in Q_{0}=D \times\left[0, t_{0}\right)$ if it satisfies Eq. (11) and verifies the initial given data (9) and prescribed conditions to the limit (10).

\section{Main results}

We assume that our micropolar porous material occupies a properly regular and bounded domain $D$ from $R^{3}$, which is the usual Euclidean space.

The set of all scalar functions defined on the domain $D$, which have a derivative of order $n$ in all points of $D$ and this derivative is a continuous function on the space $\bar{D}$, are denoted by $C^{n}(\bar{D})$.

The space $C^{n}(\bar{D})$ has the following norm:

$$
\|f\|_{C^{n}(\bar{D})}=\sum_{p=0}^{n} \sum_{j_{1}, j_{2}, \ldots, j_{p}} \max \left|f_{, j_{1} j_{2} \ldots j_{p}}\right|, \quad \forall f \in C^{n}(\bar{D}) .
$$

We denote by $\mathbf{C}^{n}(\bar{D})$ the set of vector functions with seven components, each component is a function from $C^{n}(\bar{D})$. The space $\mathbf{C}^{n}(\bar{D})$ will be endowed by the usual norm:

$$
\|v\|_{\mathbf{C}^{n}(\bar{D})}=\sum_{k=1}^{7}\left\|v_{k}\right\|_{C^{n}(\bar{D})} .
$$

The completion of the space $C^{n}(\bar{D})$ throughout the norm $\|\cdot\|_{W_{n}(D)}$ will be denoted by $W_{n}(D)$. Clearly, $W_{n}(D)$ is a Hilbert space because the norm $\|\cdot\|_{W_{n}(D)}$ is induced by the 
scalar product:

$$
(\varphi, \psi)_{W_{n}(D)}=\sum_{p=0}^{n} \int_{D} \varphi_{, j_{1} j_{2} \ldots j_{k}} \psi_{, j_{1} j_{2} \ldots j_{k}} d V .
$$

As a consequence, the completion of the space $\mathbf{C}^{n}(\bar{D})$ throughout the norm $\|\cdot\|_{\mathbf{W}_{n}(D)}$ will be denoted by $\mathbf{W}_{n}(D)$. We can deduce that $\mathbf{W}_{n}(D)$ is a Hilbert space because the norm $\|\cdot\|_{\mathbf{W}_{n}(D)}$ is induced by the scalar product

$$
(v, w)_{\mathbf{W}_{n}(D)}=\sum_{k=1}^{7}\left(v_{j}, w_{j}\right)_{W_{n}(D)} .
$$

We took into consideration that in the case of a product of normed spaces, the norm of this product is obtained by summing the norms of each factor space.

The class of all functions $f:\left[0, t_{0}\right) \rightarrow B$ having the time derivatives up to $n$th order, the last derivative being continuous on $\left[0, t_{0}\right)$, is denoted by $C^{n}\left(\left[0, t_{0}\right) ; D\right)$, in which $B$ is the notation for a Banach space.

Analog definitions can be given for the spaces $L_{1}\left(\left(0, t_{0}\right) ; D\right)$ and $L_{2}\left(\left(0, t_{0}\right) ; D\right)$ due to the clear meaning of $L_{1}$ and $L_{2}$ notations.

Definition 1 The following notations will be useful in what follows:

$$
\begin{aligned}
\hat{C}^{1}(D)= & \left\{\theta \text { of class } C^{1} \text { on } \bar{D}: \theta=0 \text { on } \partial \bar{D}_{4}\right\}, \\
\hat{\mathbf{C}}^{1}(D)= & \left\{v=(\mathbf{u}, \boldsymbol{\varphi}, \phi) \text { of class } \mathbf{C}^{1} \text { on } \bar{D}: u_{i}=0 \text { on } \partial \bar{D}_{1},\right. \\
& \left.\varphi_{i}=0 \text { on } \partial \bar{D}_{2}, \phi=0 \text { on } \partial \bar{D}_{3}\right\}, \\
\hat{W}_{1}(D)=\text { the completion of the space } \hat{C}^{1}(D) \text { throughout the norm }\|\cdot\| W_{1}(D), & \hat{\mathbf{W}}_{1}(D)=\text { the completion of the space } \hat{\mathbf{C}}^{1}(B) \text { throughout the norm }\|\cdot\| \mathbf{W}_{1}(D) .
\end{aligned}
$$

Corresponding to the function pairs $(\mathbf{u}, \varphi, \phi) \in \hat{\mathbf{C}}^{1}(D),(\mathbf{v}, \boldsymbol{\psi}, \chi) \in \hat{\mathbf{C}}^{1}(D)$, we can introduce the following functional:

$$
\begin{aligned}
F_{1}((\mathbf{u}, \boldsymbol{\varphi}, \phi),(\mathbf{v}, \boldsymbol{\psi}, \chi)) \\
=\frac{1}{2} \int_{D}\left\{A_{i j m n} \varepsilon_{m n}(\mathbf{u}, \boldsymbol{\varphi}) \varepsilon_{i j}(\mathbf{v}, \boldsymbol{\psi})\right. \\
\quad+B_{i j m n}\left[\varepsilon_{m n}(\mathbf{u}, \boldsymbol{\varphi}) \gamma_{i j}(\mathbf{v}, \boldsymbol{\psi})+\varepsilon_{m n}(\mathbf{v}, \boldsymbol{\psi}) \gamma_{i j}(\mathbf{u}, \boldsymbol{\varphi})\right]+C_{i j m n} \gamma_{m n}(\boldsymbol{\varphi}) \gamma_{i j}(\boldsymbol{\psi}) \\
\quad+a_{i j} \phi_{, j} \chi_{, i}+d_{i j k}\left[\phi_{, k} \varepsilon_{i j}(\boldsymbol{\psi})+\chi_{, k} \varepsilon_{i j}(\boldsymbol{\varphi})\right] \\
\left.\quad+e_{i j k}\left[\phi_{, k} \gamma_{i j}(\mathbf{v}, \boldsymbol{\psi})+\chi_{, k} \gamma_{i j}(\mathbf{u}, \boldsymbol{\varphi})\right]\right\} d V .
\end{aligned}
$$

Also, for the pair of functions $(T, \theta)$, we define the functional

$$
F_{2}(T, \theta)=\int_{D} K_{i j} \theta_{, i} T_{, j} d V
$$


We can extend by continuity the functionals $F_{1}$ and $F_{2}$ to the space $\hat{\mathbf{W}}_{1}(D)$ and, respectively, to space $\hat{W}_{1}(D)$.

In order to obtain our results, we need the following hypotheses with regard to the material properties:

(i) $\varrho>0, \quad I_{i j}, \kappa>0, \quad a>0, \quad T_{0}>0$;

(ii) the functionals $F_{1}$ and $F_{2}$ must satisfy the inequalities

$$
\begin{aligned}
& F_{1}((\mathbf{v}, \boldsymbol{\psi}, \chi),(\mathbf{v}, \boldsymbol{\psi}, \chi)) \\
& \quad \geq c_{1} \int_{D} \sum_{m, n=1}^{3}\left[\varepsilon_{m n}^{2}((\mathbf{v}, \boldsymbol{\psi}, \chi),(\mathbf{v}, \boldsymbol{\psi}, \chi))+\gamma_{m n}^{2}((\mathbf{v}, \boldsymbol{\psi}, \chi),(\mathbf{v}, \boldsymbol{\psi}, \chi))+\chi, i \chi, j\right] d V
\end{aligned}
$$

for any $((\mathbf{v}, \boldsymbol{\psi}, \chi),(\mathbf{v}, \boldsymbol{\psi}, \chi)) \in \hat{\mathbf{C}}^{1}(D)$, where $c_{1}$ is a positive constant;

$$
F_{2}(\theta, \theta) \geq c_{2} \int_{D} K_{i j} \theta_{, i} \theta_{, j} d V
$$

for any $\theta \in \hat{C}^{1}(D)$, where $c_{2}$ is a positive constant.

Clearly, inequality (15) can be generalized for $(\mathbf{v}, \boldsymbol{\psi}, \chi),(\mathbf{v}, \boldsymbol{\psi}, \chi) \in \hat{\mathbf{W}}_{1}(D)$ and inequality (16) can be generalized for $\theta \in \hat{W}_{1}(D)$.

According to [22, 23], we can find a positive constant $k$ such that the following inequality takes place:

$$
\begin{aligned}
& F_{1}((\mathbf{v}, \boldsymbol{\psi}, \chi),(\mathbf{v}, \boldsymbol{\psi}, \chi)) \\
& \quad \geq k \int_{D}\left[v_{i} v_{i}+\varphi_{i} \varphi_{i}+\psi_{, i} \psi_{, i}+v_{i, j} v_{i, j}+\varphi_{i, j} \varphi_{i, j}+\psi_{, i j} \psi_{, i j}\right] d V .
\end{aligned}
$$

Based on inequality (17), we obtain that the functional $F_{1}((\mathbf{v}, \psi, \chi),(\mathbf{v}, \psi, \chi))$ is coercive on the space $\hat{\mathbf{W}}_{1}(D)$.

In what follows the sets that we define now will be useful:

$$
\begin{aligned}
\tilde{C}^{0}(D)= & \left\{\left(f_{i}, g_{i}, l, r\right):\left(f_{i}, g_{i}, l\right) \in \mathbf{C}^{0}(\bar{D}), r \in C^{0}(\bar{D}) ;\right. \\
& \text { if } \operatorname{meas}\left(\partial \bar{D}_{1}\right)=\operatorname{meas}\left(\partial \bar{D}_{2}\right)=\operatorname{meas}\left(\partial \bar{D}_{3}\right)=0 \\
& \Rightarrow \int_{D} \varrho F d V=0, \int_{D} \varrho(x \times F) d V=0 ; \\
& \text { if meas }\left(\partial \bar{D}_{1}\right)=0, \operatorname{meas}\left(\partial \bar{D}_{2}\right)=0, \operatorname{meas}\left(\partial \bar{D}_{3}\right) \neq 0, \Rightarrow \int_{D} \varrho F d V=0 ; \\
& \text { if } \left.\operatorname{meas}\left(\partial \bar{D}_{4}\right)=0, \Rightarrow \int_{D} \varrho r d V=0\right\} ; \\
\tilde{C}^{1}(D)= & \left\{\left(u=\left(u_{i}, \varphi_{i}, \phi\right), \theta\right):(u, \theta) \in \hat{\mathbf{C}}^{1}(D) \times \hat{C}^{1}(D)\right. \text { and } \\
& \text { if } \left.\operatorname{meas}\left(\partial \bar{D}_{4}\right)=0 \Rightarrow \int_{D}\left[\alpha_{i j} \varepsilon_{i j}(u)+\beta_{i j} \gamma_{i j}(u)+a \theta+a_{i} \phi_{, i}\right] d V=0\right\} ;
\end{aligned}
$$




$$
\begin{aligned}
& \mathcal{D}\left(Q_{0}\right)=C^{\infty}\left(\left[0, t_{0}\right) ; \tilde{C}^{1}(D)\right) ; \\
& \breve{\mathcal{D}}\left(Q_{0}\right)=\left\{\left(v=\left(v_{i}, \psi_{i}, \chi\right), T\right):(v, T) \in \mathcal{D}\left(Q_{0}\right) \text { and } v=0 \text { on } D \times\{0\}\right\} .
\end{aligned}
$$

For the elements $y=\left(\left(u_{i}, \varphi_{i}, \phi\right), \theta\right) \in \mathcal{D}\left(Q_{0}\right), w=\left(\left(v_{i}, \psi_{i}, \chi\right), T\right) \in \breve{\mathcal{D}}\left(Q_{0}\right)$ and considering the charges $z=\left(f_{i}, g_{i}, r\right) \in \mathbf{C}^{\infty}\left(\left[0, t_{0}\right) ; \tilde{C}^{0}(D)\right)$, we define the functionals $F_{3}(y, w)$ and $F_{4}(z, w)$ as follows:

$$
\begin{aligned}
F_{3}(y, w)= & \int_{0}^{t_{0}} \int_{D}\left\{( t - t _ { 0 } ) \left[\varrho \dot{u}_{i} \ddot{v}_{i}+I_{i j} \dot{\varphi}_{i} \ddot{\psi}_{j}+\varrho \kappa \dot{\phi} \ddot{\chi}-A_{i j m n} \varepsilon_{m n}(y) \dot{\varepsilon}_{i j}(w)\right.\right. \\
& -B_{i j m n}\left(\varepsilon_{m n}(y) \dot{\gamma}_{i j}(w)+\dot{\varepsilon}_{m n}(w) \dot{\gamma}_{i j}(y)\right)-C_{i j m n} \gamma_{m n}(y) \dot{\gamma}_{i j}(w) \\
& -D_{i j k}\left(\phi_{, k} \dot{\varepsilon}_{i j}(w)+\dot{\chi}_{, k} \varepsilon_{i j}(y)\right)-E_{i j k}\left(\phi_{, k} \dot{\gamma}_{i j}(w)+\dot{\chi}_{, k} \gamma_{i j}(y)\right) \\
& \left.+a_{i j} \phi_{, i} \dot{\chi}_{, j}+a \theta \dot{T}+\alpha_{i j} \theta \dot{\varepsilon}_{i j}(w)+\beta_{i j} \theta \dot{\gamma}_{i j}(w)+a_{i} \dot{\phi}_{, i} \theta\right] \\
& +\varrho \dot{u}_{i} \dot{v}_{i}+I_{i j} \dot{\varphi}_{i} \dot{\psi}_{j}+\varrho \kappa \dot{\phi} \dot{\chi}+a \theta T+a_{i} \varphi_{, i} T \\
& \left.+\alpha_{i j} T \varepsilon_{i j}(y)+\beta_{i j} T \gamma_{i j}(y)+\frac{1}{T_{0}} \int_{0}^{t} K_{i j} \theta_{, j} T_{, i} d \tau\right\} d V d t \\
F_{4}(z, w)= & \int_{0}^{t_{0}} \int_{B}\left(t-t_{0}\right)\left[-\varrho f_{i} \dot{v}_{i}-I_{i j} g_{i} \dot{\psi}_{j}-\varrho \kappa l \dot{\chi}-\frac{\varrho r}{T_{0}} T\right] d V d t .
\end{aligned}
$$

Also, taking into account the initial data $\delta=\left(u_{i}^{0}, \varphi_{i}^{0}, \phi^{0}, \theta^{0}\right)$ such that $u_{i}^{0}, \varphi_{i}^{0} \in \hat{\mathbf{C}}^{1}(D)$, $\phi^{0}, \theta^{0} \in \tilde{C}^{1}(D)$, we will consider the functional

$$
\begin{aligned}
F_{5}(\delta, w)= & t_{0} \int_{D}\left[\left.\varrho u_{i}^{0} v_{i}\right|_{t=0}+\left.I_{i j} \varphi_{i}^{0} \psi_{j}\right|_{t=0}+\left.\varrho \kappa \chi\right|_{t=0} \phi^{0}+\left.a T\right|_{t=0} \theta^{0}\right. \\
& \left.+\left.\alpha_{i j} T\right|_{t=0} \varepsilon_{i j}\left(u^{0}, \theta^{0}\right)+\left.\beta_{i j} T\right|_{t=0} \gamma_{i j}\left(u^{0}, \theta^{0}\right)+\left.a_{i} T\right|_{t=0} \varphi_{, i}\right] d V .
\end{aligned}
$$

If we replace $y$ by $w$ in $F_{3}(z, w)$, then we obtain the following identity:

$$
\begin{aligned}
F_{3}(w, w)= & \int_{0}^{t_{0}} \int_{D}\left(t-t_{0}\right)\left[\varrho \dot{v}_{i} \dot{v}_{i}+I_{i j} \dot{\psi}_{i} \dot{\psi}_{j}+\varrho \kappa \dot{\chi}^{2}+A_{i j m n} \varepsilon_{m n}(w) \varepsilon_{i j}(w)\right. \\
& +2 B_{i j m n} \varepsilon_{m n}(w) \gamma_{i j}(w)+C_{i j m n} \gamma_{m n}(w) \dot{\gamma}_{i j}(w) \\
& +2 D_{i j k} \chi_{, k} \varepsilon_{i j}(w)+2 E_{i j k} \chi_{, k} \gamma_{i j}(w)+a_{i j} \chi_{, i} \chi_{, j}+a T^{2} \\
& \left.+\frac{1}{T_{0}} \int_{0}^{t} K_{i j} \theta_{, j} T_{, i} d \tau\right] d V d t \\
& +\frac{t_{0}}{2} \int_{D}\left[\varrho \dot{v}_{i} \dot{v}_{i}+I_{i j} \psi_{i} \psi_{j}+\varrho \kappa \chi^{2}+a T^{2}\right]_{t=0} d V .
\end{aligned}
$$

Our goal is to find a solution with finite energy for the mixed problem constituted by Eq. (11), the initial conditions (9), and the boundary conditions (10). To this end, we need some new spaces. 
First, we consider the completion of the space $\mathcal{D}\left(Q_{0}\right)$ throughout the norm $|\cdot|$ as being the Hilbert space $V\left(Q_{0}\right)$, the norm $|\cdot|$ being induced by the following scalar product:

$$
\begin{aligned}
& \left\langle\left(u_{i}, \varphi_{i}, \phi, \theta\right),\left(v_{i}, \psi_{i}, \chi, T\right)\right\rangle \\
& =\int_{0}^{t_{0}} \int_{D}\left[u_{i} \dot{v}_{i}+\varphi_{i} \dot{\psi}_{i}+\phi \dot{\chi}\right. \\
& \left.\quad+\dot{u}_{i} \dot{v}_{i}+\dot{\varphi}_{i} \dot{\psi}_{i}+\dot{\phi} \dot{\chi}+u_{i, j} v_{i, j}+\varphi_{i, j} \psi_{i, j}+\phi_{, i} \chi_{, i}+\theta T+\int_{0}^{t} \theta_{, i} T_{, i}\right] d V d t .
\end{aligned}
$$

Also, we denote by $\breve{V}\left(Q_{0}\right)$ the completion of the space $\breve{\mathcal{D}}\left(Q_{0}\right)$ obtained by means of the above norm $|\cdot|$. Let us observe that $\breve{V}\left(Q_{0}\right)$ is a closed linear manifold.

Another Hilbert space is defined as the completion of the space $\breve{\mathcal{D}}\left(Q_{0}\right)$ throughout the norm which is generated by the following scalar product:

$$
\begin{aligned}
& {\left[\left(u_{i}, \varphi_{i}, \phi, \theta\right),\left(v_{i}, \psi_{i}, \chi, T\right)\right]} \\
& \quad=\left\langle\left(u_{i}, \varphi_{i}, \phi, \theta\right),\left(v_{i}, \psi_{i}, \chi, T\right)\right\rangle+\left\langle\left(\dot{u}_{i}, \dot{\varphi} i, \dot{\phi}, \dot{\theta}\right),\left(\dot{v}_{i}, \dot{\psi}_{i}, \dot{\chi}, \dot{T}\right)\right\rangle,
\end{aligned}
$$

where the product $\langle(u, \theta),(v, T)\rangle$ was already defined in (19). This Hilbert space will be denoted by $\breve{U}\left(Q_{0}\right)$.

Also, the space $G(D)$ is obtained as a completion of $\tilde{C}^{0}(D)$ throughout the norm $\|\cdot\|_{\mathbf{W}_{0}(D) \times W_{0}(D)}$. At last, the completion of the set

$$
\left\{(u, v, \theta):\left(u=\left(u_{i}, \varphi_{i}, \phi\right), \theta\right) \in \tilde{\mathbf{C}}^{1}(D),\left(v=\left(v_{i}, \psi_{i}, \chi\right), \theta\right) \in \hat{\mathbf{C}}^{1}(D)\right\},
$$

throughout the norm

$$
\begin{aligned}
|(u, v, \theta)|_{0}= & \left\{\frac { 1 } { 2 } \int _ { D } \left[\varrho v_{i} v_{i}+I_{i j} \psi_{i} \psi_{j}+\varrho \kappa \chi^{2}+A_{i j m n} \varepsilon_{i j}(u) \varepsilon_{m n}(v)\right.\right. \\
& +2 B_{i j m n} \varepsilon_{i j}(u) \gamma_{m n}(v)+C_{i j m n} \gamma_{i j}(u) \gamma_{m n}(v)+2 D_{i j k} e_{i j}(u) \varphi_{, k} \\
& \left.\left.+2 E_{i j k} \gamma_{i j}(u) \chi_{, k}+a_{i j} \phi_{, i} \chi_{, j}+a \theta^{2}\right] d v\right\}^{1 / 2},
\end{aligned}
$$

will be denoted by $H_{0}(B)$.

With the help of Sobolev's embedding theorem and by using Schwarz's inequality, we can extend by continuity the functional $F_{3}(y, w)$ on the product space $\breve{U}\left(Q_{0}\right) \times V\left(Q_{0}\right)$ and, analogously, $F_{4}(z, \delta)$ can be an extended functional on the space $\breve{U}\left(Q_{0}\right) \times L_{1}\left(\left(0, t_{0}\right) ; G(D)\right)$.

Similarly, we can extend $F_{5}(\delta, w)$ so that it can exist for $\delta \in H_{0}(D), w \in \breve{U}\left(Q_{0}\right)$.

Taking into account the above completions and extensions and considering the hypotheses (14)-(17) and identity (18), we can find a positive constant $c_{3}$, depending only on $T_{0}$, $k$, and $a$, to have the estimation

$$
|w|^{2} \leq c_{3} F_{3}(w, w), \quad \forall w \in \breve{U}\left(Q_{0}\right) .
$$

Consider $u=\left(u_{i}, \varphi_{i}, \phi\right)$ and $y \equiv(u, \theta) \in V\left(Q_{0}\right), y$ satisfies our mixed problem consisting of the system of equations (11), the data to the limit conditions (10), and the initial data (9). 
Below, we can define the concept of solution, having a finite energy, in the case of the mixed problem defined in our context.

Definition 2 If $y=(u, \theta)$ satisfies the conditions

$$
\begin{aligned}
& F_{3}(y, w)=F_{4}(z, w)+F_{5}(\delta, w), \quad \forall w=\left(\left(v_{i}, \psi_{i}, \chi\right), T\right) \in \breve{U}\left(Q_{0}\right), \\
& \lim _{t \rightarrow 0} u(t)=u_{0}, \quad \text { in } \mathbf{W}_{0}(D),
\end{aligned}
$$

then $y$ is called the finite energy solution that satisfies Eq. (11) and verifies the conditions to the limit (10) and corresponds to the initial data $\delta \equiv\left(u_{i}^{0}, \varphi_{i}^{0}, \phi^{0}, \theta^{0}\right) \in \mathbf{H}_{0}(D)$, having the loads $z \equiv(\mathbf{f}, \mathbf{g}, l, r) \in \mathbf{L}_{1}\left(\left(0, t_{0}\right) ; G(D)\right)$.

The result that follows shows a way to find a new finite energy solution, starting from a given solution.

Theorem 1 Consider y to be a solution with finite energy, $y \in V\left(Q_{0}\right)$, which verifies Eq. (11) and satisfies the conditions to the limit (10), defined on the cylinder $Q_{0}$, and which corresponds to the source $z=\left(f_{i}, g_{i}, l, r\right)$ and to the initial conditions $\delta \equiv\left(u_{i}^{0}, \varphi_{i}^{0}, \phi^{0}, \theta^{0}\right)$.

Define the function $\tilde{y}$ by

$$
\breve{y}=\int_{0}^{t} y(x, s) d s .
$$

Then $\breve{y}$ is also a solution with finite energy for problem (11), (10) defined in cylinder $Q_{0}$, which corresponds to the initial conditions $\breve{\delta}=\left(0,0, \phi^{0}, 0\right)$ and to the source $\breve{z}=\left(\breve{f}_{i}, \breve{g}_{i}, \breve{l}, \breve{r}\right)$, in which we used the notations

$$
\begin{aligned}
\breve{f}_{i}(x, t)= & \int_{0}^{t} f_{i}(x, s) d s+v_{i}^{0}(x), \\
\breve{g}_{i}(x, t)= & \int_{0}^{t} g_{i}(x, s) d s+\psi_{i}^{0}(x), \\
\breve{l}_{i}(x, t)= & \int_{0}^{t} l(x, s) d s+\chi^{0}(x), \\
\breve{r}_{i}(x, t)= & \int_{0}^{t} r(x, s) d s \\
& +\frac{T_{0}}{\varrho}\left[\alpha_{i j}(x) \varepsilon_{i j}\left(u^{0}\right)+\beta_{i j}(x) \gamma_{i j}\left(u^{0}\right)+a(x) \theta^{0}+a_{i}(x) \phi_{, i}^{0}\right] .
\end{aligned}
$$

Proof The result can be obtained by using the same procedure as in Dafermos [26].

Also, by using a procedure similar to the one used in Dafermos [26], we can prove the following uniqueness result with regard to the finite energy solution of the mixed problem.

Theorem 2 In thermoelasticity of initially stressed bodies with voids, the mixed problem consisting of system (11) and the data to the limit (10) admits only one finite energy solution which corresponds to some given initial conditions and some given sources. 
Let us restrict the considerations to the more simple case of null initial data, the sources belonging to $L_{2}\left(\left(0, t_{0}\right) ; G(D)\right)$. In this case, we have the open path to demonstrate the existence result for a solution with finite energy as well as an estimate of this solution. These results are included in the following theorem.

Theorem 3 In thermoelasticity of initially stressed bodies with pores, we consider the mixed problem consisting of system (11) and the boundary data (10) which corresponds to the source $z=\left(\left(f_{i}, g_{i}, l\right), r\right) \in \mathbf{L}_{2}\left(\left(0, t_{0}\right) ; G(D)\right)$ and to null initial data. Then this problem admits at least a finite energy solution $y=\left(u_{i}, \varphi_{i}, \phi, \theta\right) \in \breve{V}\left(Q_{0}\right)$. Furthermore, we can determine a positive constant $c_{4}$, depending on $c_{3}($ from $(20)), \varrho, T_{0}$, and $t_{0}$, so that the following estimation takes place:

$$
|y| \leq c_{4}\|z\|_{\mathbf{L}_{2}\left(Q_{0}\right) \times L_{2}\left(Q_{0}\right)} .
$$

Now, we want to extend the result of the existence for the solution with finite energy from the particular case of the null initial data from Theorem 2 to the case of some arbitrary initial data. To this aim we need the following definitions.

Definition 3 We define the Hilbert space $H(D)$ by

$$
H(D)=H_{0}(D) \cap\left\{\hat{\mathbf{W}}_{1}(D) \times \hat{\mathbf{W}}_{1}(D) \times \hat{W}_{1}(D)\right\} .
$$

If the source $z=\left(\left(f_{i}, g_{i}, l\right), r\right) \in G(B)$, then the map $\mathcal{S}(z): H_{0}(D) \rightarrow H(D)$ that made the transfer $\left(u_{i}, \psi_{i}, \chi, T\right) \in H_{0}(D)$ into $\left(u_{i}, \varphi_{i}, \phi, \theta\right) \in H(D)$, where $\left(u_{i}, \varphi_{i}, \phi, \theta\right) \in \hat{\mathbf{W}}_{1}(D) \times$ $\hat{W}_{1}(D)$, will be named as solution for the following system:

$$
\begin{aligned}
F_{1}(u, \omega)= & \int_{D}\left[\left(\alpha_{i j} \varepsilon_{i j}(\omega)+\beta_{i j} \gamma_{i j}(\omega)+a_{i} \psi_{, i}+a T\right) \theta\right. \\
& \left.-\varrho u_{i} v_{i}-I_{i j} \varphi_{i} \psi_{j}-\varrho \kappa \chi+\varrho f_{i} v_{i}+\varrho g_{i} \psi_{i}+\varrho l \chi\right], \\
& \forall \omega=\left(u_{i}, \psi_{i}, \chi, T\right) \in \hat{\mathbf{W}}_{1}(D), \\
F_{2}(T, \theta)= & -\int_{D}\left\{T_{0}\left[\alpha_{i j} \varepsilon_{i j}(u)+\beta_{i j} \gamma_{i j}(u)+a_{i} \psi_{, i}+a T\right]-\varrho r\right\} \theta d V, \quad \forall T \in \hat{W}_{1}(D) .
\end{aligned}
$$

In the following theorem we characterize the solution defined by means of the map $\mathcal{S}(z)$ in Theorem 3. Also, the smoothness of this kind solution is investigated, taking into account some hypotheses on the initial data and some specific sources. This is an auxiliary step to obtain the existence of the solution in the general case of the initial conditions.

Theorem 4 For any $z \in H_{0}(D), \mathcal{S}(z)$ is a well-defined and one-to-one map so that the inverse map $\mathcal{S}^{-1}(z)$ is defined for all $z$ from the codomain of $\mathcal{S}(z) \subset H(D)$ to $H_{0}(D)$.

Also, this solution satisfies the following estimation with regard to the general initial data $\delta=\left(u_{i}^{0}, \varphi_{i}^{0}, \phi^{0}, \theta^{0}\right)$ and the sources $z=\left(f_{i}, g_{i}, l, r\right)$ :

$$
\|\mathcal{S}(z)\|_{\mathbf{W}_{1}(D) \times \mathbf{W}_{1}(D) \times W_{1}(D)} \leq c_{5}\left\{|\delta|_{0}+\|z\|_{\mathbf{w}_{0}(D) \times W_{0}(D)}\right\},
$$

where $c_{5}$ is a positive constant. 
Proof To prove our result, we can use the same procedure as Fischera in the paper [25]. So, we must take into account that the functional $F_{1}$ satisfies inequality (15). Also, we must consider that the functional $F_{1}(v, v)$ is coercive in the space $\hat{\mathbf{W}}_{1}(D)$ with respect to the norm $\|\cdot\|_{\mathbf{w}_{1}(D)}$, which follows from inequality (17).

Let us consider some arbitrary sources $z_{0}, z_{1}, \ldots, z_{m-1}$, such that $z_{0}, z_{1}, \ldots, z_{m-1} \in G(D)$, and introduce the map

$$
\mathcal{S}_{m}\left(z_{0}, z_{1}, \ldots, z_{m-1}\right)=\mathcal{S}\left(z_{0}\right) \circ \mathcal{S}\left(z_{1}\right) \circ \cdots \circ \mathcal{S}\left(z_{m-1}\right)
$$

The codomain of the application

$$
\mathcal{S}_{m}\left(y_{0}, y_{1}, \ldots, y_{m-1}\right): H_{0}(D) \rightarrow H(D)
$$

will be denoted by $H_{m}\left(B ; y_{0}, y_{1}, \ldots, y_{m-1}\right)$. If we set $H_{m}(D)=H_{m}\left(D ; y_{0}, y_{1}, \ldots, y_{m-1}\right)$ for $\delta \in$ $H_{m}(B)$, we introduce a new norm

$$
|\delta|_{m}=\left|\mathcal{S}_{m}^{-1}(0,0, \ldots, 0) \delta\right|_{0} .
$$

The notation $\stackrel{(m)}{f}=\frac{\partial^{m} f}{\partial z_{i}^{m}}$ will be used for the partial derivative of order $m$ for a function $f=f\left(z_{1}, z_{2}, \ldots, z_{n}\right)$.

Based on these new considerations, we can address the problem of the existence of a finite energy solution in the general case of some non-homogeneous initial data.

Theorem 5 The mixed problem consisting of system (11), satisfied in $Q(0)$, the conditions to the limit (10), the charges

$$
z=\left(f_{i}, g, l, r\right) \in C^{m-1}\left(\left[0, t_{0}\right) ; G(D)\right), \quad \stackrel{(m)}{z} \in L_{1}\left(\left(0, t_{0}\right) ; G(D)\right)
$$

and the initial restrictions

$$
\delta=\left(u_{i}^{0}, \varphi_{i}^{0}, \phi^{0}, \theta^{0}\right) \in H_{m}(D ; z(0), \stackrel{(1)}{z}(0), \ldots, \stackrel{(m-1)}{z}(0)) \quad \text { for } m=1,2, \ldots
$$

admits at least one solution with finite energy $y \in V\left(Q_{0}\right)$.

Proof The result can be obtained by using the same procedure as Fischera in the paper [25].

Using the procedure of Dafermos [26] and Fichera [25], we can deduce the following three properties regarding the controllability of the finite energy solution from Theorem 5 .

Theorem 6 Any solution with finite energy of the mixed problem satisfies the following estimations:

(i) $\left(u_{i}, \varphi_{i}, \phi, \dot{u}_{i}, \dot{\varphi}_{i}, \dot{\phi}, \theta\right) \in C^{m}\left(\left[0, t_{0}\right) ; H_{0}(D)\right)$,

$$
\left.\left(u_{i}, \varphi_{i}, \phi, \dot{u}_{i}, \dot{\varphi}_{i}, \dot{\phi}, \theta\right)\right|_{(0)}=\left(u_{i}^{0}, \varphi_{i}^{0}, \phi^{0}, \dot{u}_{i}^{1}, \dot{\varphi}_{i}^{1}, \dot{\phi}^{1}, \theta^{0}\right) ;
$$


(ii) for all $k=0,1, \ldots, m$ and $t \in\left[0, t_{0}\right)$, we have

$$
\begin{aligned}
& \left.\left(^{(k)}\right)^{(k)}{ }^{(k)}{ }^{(k+1)}(k+1)^{(k+1)}{ }^{(k)}\right) \in \mathcal{S}{ }^{(k)}{ }^{(k)}{ }^{(k)+1} \quad{ }^{(m-1)} \\
& \left(u_{i}, \varphi_{i}, \phi, \stackrel{u_{i}}{u_{i}}, \stackrel{\varphi_{i}}{\varphi_{i}}, \phi, \theta\right) \in \mathcal{S}_{m-k}(D ; z(t), z(t), \ldots, z(t)) \text {, }
\end{aligned}
$$

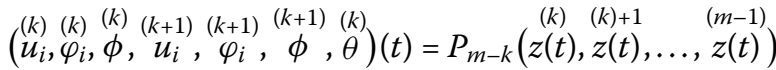

$$
\begin{aligned}
& =\left(\stackrel{(m)}{u_{i}}, \stackrel{(m)}{\varphi} \varphi_{i}, \stackrel{(m)}{\phi}, \stackrel{(m+1)}{u_{i}}, \stackrel{(m+1)}{\varphi}{ }_{i}, \stackrel{(m+1)}{\phi}, \stackrel{(m)}{\theta}\right)(t) ;
\end{aligned}
$$

(iii) we have the estimation

$$
\begin{aligned}
& \left(\left|\left(\stackrel{(k)}{u_{i}}, \stackrel{(k)}{\varphi}, \stackrel{(k)}{\phi}, \stackrel{(k+1)}{u_{i}}, \stackrel{(k+1)}{\varphi_{i}}, \stackrel{(k+1)}{\phi}, \stackrel{(k)}{\theta}\right)(t)\right|_{0}^{2}+\frac{1}{T_{0}} \int_{0}^{t} F_{2}(\stackrel{(k)}{\theta}, \stackrel{(k)}{\theta})(s) d s\right)^{1 / 2} \\
& \quad \leq\left|\mathcal{S}_{k}^{-1}(z(0), \stackrel{(1)}{z}(0), \ldots, \stackrel{(m-1)}{z}(0))\right|_{0}+c_{6} \int_{0}^{t}\|\stackrel{(k)}{z}(s)\|_{\mathbf{W}_{0}(D) \times W_{0}(D)} d s,
\end{aligned}
$$

where $c_{6}$ is a positive constant depending on $T_{0}, \varrho, I_{i j}$, and a. Also, estimation (23) takes place for all $k=0,1, \ldots, m$ and $t \in\left[0, t_{0}\right)$.

Remark It is worth noting that in the simple case of null loads, $z \equiv 0$, inequality (23) turns into equality.

\section{Conclusions}

We first put down the main equations and basic conditions for the mixed problem in the theory of micropolar thermoelastic materials with pores. Then we extended the procedure proposed by Dafermos in [26] and Fichera in [25] in order to obtain some uniqueness and existence results. The first result is the uniqueness of the finite energy solution. Then we prove the existence result for a finite energy solution in the particular case of null initial data. In Theorem 5 this result is extended in order to cover the general case of non-homogeneous initial conditions. The last theorem of our study is dedicated to some properties regarding the controllability of the finite energy solution. We need to emphasize the validity of the results regarding the uniqueness and existence for the finite energy solution is as in the classical theory, even if the context in our study is much more complicated, because we considered the effect of the micropolar structure, the effect of thermal treatment, and the effect of voids.

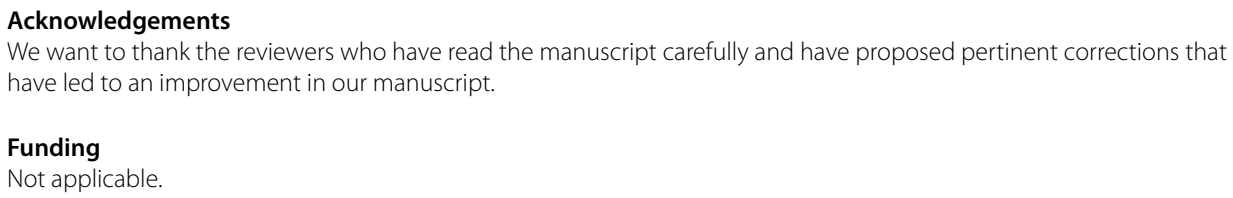




\section{Author details}

${ }^{1}$ Department of Mathematics and Computer Science, Transilvania University of Brasov, Brasov, Romania. ${ }^{2}$ Faculty of Mechanical Engineering, Esslingen University of Applied Sciences, Esslingen, Germany. ${ }^{3}$ Department of Mechanical Engineering, Transilvania University of Brasov, Brasov, Romania.

\section{Publisher's Note}

Springer Nature remains neutral with regard to jurisdictional claims in published maps and institutional affiliations.

Received: 17 April 2019 Accepted: 6 May 2019 Published online: 14 May 2019

\section{References}

1. Goodman, M.A., Cowin, S.C.: A continuum theory of granular material. Arch. Ration. Mech. Anal. 44, $249-266$ (1972)

2. Cowin, S.C., Nunziato, J.W.: Linear elastic materials with voids. J. Elast. 13, 125-147 (1983)

3. Nunziato, J.W., Cowin, S.C.: A nonlinear theory of materials with voids. Arch. Ration. Mech. Anal. 72, 175-201 (1979)

4. Iesan, D.: A theory of thermoelastic material with voids. Acta Mech. 60, 67-89 (1986)

5. Eringen, A.C.: Theory of thermo-microstretch elastic solids. Int. J. Eng. Sci. 28, 1291-1301 (1990)

6. Eringen, A.C.: Nonlocal Continuum Field Theories. Springer, New York (2002)

7. Iesan, D., Quintanilla, R.: On the thermoelastic bodies with inner structure and microtemperatures. J. Math. Anal. Appl. $354,12-23(2009)$

8. Svanadze, M.: On the linear equilibrium theory of elasticity for materials with triple voids. Q. J. Mech. Appl. Math. 71(3), 329-348 (2018)

9. Marin, M.: Cesaro means in thermoelasticity of dipolar bodies. Acta Mech. 122(1-4), 155-168 (1997)

10. Abbas, I.A.: A GN model based upon two-temperature generalized thermoelastic theory in an unbounded medium with a spherical cavity. Appl. Math. Comput. 245, 108-115 (2014)

11. Abbas, I.A.: Eigenvalue approach for an unbounded medium with a spherical cavity based upon two-temperature generalized thermoelastic theory. J. Mech. Sci. Technol. 28(10), 4193-4198 (2014)

12. Abbas, I.A., Abo-Dahab, S.M.: On the numerical solution of thermal shock problem for generalized magneto-thermoelasticity for an infinitely long annular cylinder with variable thermal conductivity. J. Comput. Theor. Nanosci. 11(3), 607-618 (2014)

13. Othman, M.I.A.: State space approach to generalized thermoelasticity plane waves with two relaxation times under the dependence of the modulus of elasticity on reference temperature. Can. J. Phys. 81(12), 1403-1418 (2003)

14. Sharma, J.N., Othman, M.I.A.: Effect of rotation on generalized thermo-viscoelastic Rayleigh-Lamb waves. Int. J. Solids Struct. 44(13), 4243-4255 (2007)

15. Othman, M.I.A., Hasona, W.M., Abd-Elaziz, E.M.: Effect of rotation on micropolar generalized thermoelasticity with two-temperatures using a dual-phase-lag model. Can. J. Phys. 92(2), 149-158 (2014)

16. Marin, M., Öchsner, A.: The effect of a dipolar structure on the Holder stability in Green-Naghdi thermoelasticity. Contin. Mech. Thermodyn. 29(6), 1365-1374 (2017)

17. Hassan, M., Marin, M., Alsharif, A., Ellahi, R.: Convective heat transfer flow of nanofluid in a porous medium over wavy surface. Phys. Lett. A 382(38), 2749-2753 (2018)

18. Marin, M., Nicaise, S.: Existence and stability results for thermoelastic dipolar bodies with double porosity. Contin. Mech. Thermodyn. 28(6), 1645-1657 (2016)

19. Marin, M., Vlase, S., Carstea, C.: A dipolar structure in the heat-flux dependent thermoelasticity. AIP Adv. 8, 035220 (2018)

20. Modrea, A., Vlase, S., et al.: The influence of dimensional and structural shifts of the elastic constant values in cylinder fiber composites. J. Optoelectron. Adv. Mater. 15(3-4), 278-283 (2013)

21. Niculita, C., Vlase, S., et al.: Optimum stacking in a multi-ply laminate used for the skin of adaptive wings. J. Optoelectron. Adv. Mater. 5(11), 1233-1236 (2011)

22. Craciun, E.M., Barbu, L.: Compact closed form solution of the incremental plane states in a pre-stressed elastic composite with an elliptical hole. Z. Angew. Math. Mech. 95(2), 193-199 (2015)

23. Marin, M., Craciun, E.M., Pop, N.: Considerations on mixed initial-boundary value problems for micropolar porous bodies. Dyn. Syst. Appl. 25(1-2), 175-196 (2016)

24. Marin, M., Radulescu, V.: A variational approach for the mixed problem in the elastostatics of bodies with dipolar structure. Mediterr. J. Math. 15(6), 221 (2018)

25. Fichera, G.: Existence theorems in elasticity. In: Truesdell, C. (ed.) Linear Theories of Elasticity and Thermoelasticity, vol. VI a/2, pp. 347-424. Springer, Berlin (1973)

26. Dafermos, C.M.: On the existence and the asymptotic stability of solutions to the equations of linear thermoelasticity. Arch. Ration. Mech. Anal. 29(4), 241-271 (1968)

27. Visik, M.I.: Quasi-linear strongly elliptic systems of differential equations in divergence form. Trans. Mosc. Math. Soc. $12,140-208(1963)$

28. Carbonaro, B., Russo, R.: Energy inequalities in classical elastodynamics. J. Elast. 14, 163-174 (1984)

29. Marin, M., Öchsner, A.: Essentials of Partial Differential Equations with Applications. Springer, Cham (2019) 